\title{
Parasitismo por malófagos (Insecta) e ácaros (Acari) em Turdus leucomelas (Aves) nas estações reprodutiva e de muda de penas no Parque Estadual do Rio Preto, Minas Gerais, Brasil
}

\author{
Alexandre M. J. Enout ${ }^{\text {; }}$, Débora N. Lobato ${ }^{2}$; Cristiano S. de Azevedo ${ }^{3} \&$ Yasmine Antonini ${ }^{1}$ \\ ${ }^{1}$ Laboratório de Biodiversidade, Departamento de Ciências Biológicas, Instituto de Ciências Exatas e Biológicos, Universidade \\ Federal de Ouro Preto. Campus Morro do Cruzeiro, Bauxita, 35400-000 Ouro Preto, Minas Gerais, Brasil. \\ E-mail: aleenout@hotmail.com \\ ${ }^{2}$ Laboratório de Ecologia e Comportamento de Insetos, Departamento de Biologia Geral, Instituto de Ciências Biológicas, \\ Universidade Federal de Minas Gerais. Avenida Antônio Carlos 6627, Caixa Postal 486, 31270-901 Belo Horizonte, Minas \\ Gerais, Brasil. E-mail: antonini@iceb.ufop.br \\ ${ }^{3}$ Laboratório de Ornitologia, Departamento de Zoologia, Instituto de Ciências Biológicas, Universidade Federal de Minas \\ Gerais. Avenida Antônio Carlos 6627, 31270-901 Belo Horizonte, Minas Gerais, Brasil. E-mail: cristianoroxette@yahoo.com
}

\begin{abstract}
Parasitism of chewing lice (Insecta) and feather mites (Acari) on Turdus leucomelas (Aves) during reproductive and molt seasons at the State Park of Rio Preto, Minas Gerais, Brazil. The rates of infestation by chewing lice and feather mites on Turdus leucomelas Vieillot, 1818 (Aves: Turdidae) were used to test the hypothesis of that parasitism either increases or decreases according to, reproductive and molting seasons, respectively. Samples were obtained at the State Park of Rio Preto, state of Minas Gerais, Brazil, in October and December of 2006 and in March of 2007. The dustruffling technique was used in to remove the parasites from the body of their hosts. Five species of chewing-lice were found on the birds, with Myrsidea sp. and Brueelia sp. being the most prevalent parasites, whereas Menacanthus eurysternus Burmeister, 1838 was the least prevalent. No statistically significant difference was found between the infestation rates in the reproductive and molt seasons. Two suborders and four families of Acari were found on the birds.
\end{abstract}

KEY WORDS. Chewing lice; feather mites; feather molt; pyrethrin.

Embora o conhecimento sobre os parasitos de aves de produção seja relativamente extenso, estudos sobre parasitos de aves silvestres neotropicais ainda são restritos. Estudos relativos à taxonomia de malófagos e ácaros são os mais comuns e novas espécies têm sido descritas recentemente (e.g. VALIM \& HeRnANDES 2006, Hernandes \& Valim 2005, 2006, Chicchino \& Valim 2008). Contudo, existem alguns exemplos de trabalhos que enfocam a relação dos ectoparasitos com seus hospedeiros como o trabalho de KANEgaE et al. (2008) que trabalharam com aves do cerrado e registraram ocorrências de ácaros de pena em novos hospedeiros. Estudos com enfoque ecológico foram realizados no Brasil, no estado de Minas Gerais, com aves de floresta e cerrado (MARINI \& Couto 1997). Na Mata Atlântica, foram realizados estudos sobre ectoparasitos associados a Turdus albicollis Vielillot, 1818 (STORNi et al. 2005); a Passeriformes da zona da mata do estado do Pernambuco (Roda \& Farias 2007) e, na região sul do Brasil, foram correlacionadas taxas de prevalência de ectoparasitos com características ecológicas das aves hospedeiras (MARINI et al. 1996). Outros estudos foram conduzidos sobre a relação de parasitismo para um hospedeiro específico, Ramphocelus carbo Pallas, 1764, e para grupos como os Emberezidae (Lrra-Neves et al 2005).
Ectoparasitos de aves são artrópodes que vivem em associação com seus hospedeiros, podendo ocorrer esporadicamente por uma fase ou por todo o seu ciclo vital. Os mais comuns são os aracnídeos, sub-classe Acari (ácaros de pena), e insetos, ordem Phthiraptera (malófagos) (Marshall 1981), os quais são ectoparasitos permanentes e obrigatórios, isto é, completam todo seu ciclo de vida no corpo do hospedeiro (JoHnson \& Clayton 2003). A permanência obrigatória nas aves durante todo ciclo de vida é, normalmente, causa da alta especificidade destes grupos de ectoparasitos e sugere forte processo de co-evolução (Paterson \& Gray 1997).

Estudos sugerem efeitos maléficos do parasitismo em aves, como abandono de ninhos e ninhegos (DufFy 1983, ClaYTON \& Moore 1997), aumento da provisão de alimento para ninhegos (PERrins 1965), incorporação de folhas com propriedades inseticidas nos ninhos (CLARK 1991), e recusa em nidificar em locais onde os parasitos são abundantes (EMlen 1985, Loye \& Carrol 1991). A resposta a estes efeitos sugere que o parasitismo é altamente custoso em termos de perda de energia e mortalidade, portanto estes custos devem ser considerados em relação ao manejo conservacionista (Loye \& CARroll 1998). 
A presença de hormônios reprodutivos no sangue das aves parece funcionar como um sinalizador para o início da reprodução dos ectoparasitos, aumentando a possibilidade de transmissão vertical; esta hipótese, embora mereça ser testada, sugere a sincronização nos ciclos de reprodução entre hospedeiros e parasitos (Marshall 1981). Outra hipótese bastante discutida prediz que durante a estação de muda das penas ocorre uma redução na abundância de ectoparasitos (MARSHALl 1981, Lehane 1991). Entretanto, Moyer et al. (2002) testaram pressupostos desta hipótese simulando a queda das penas de Columba livia Gmelin, 1789 (Columbidae) e os resultados não apontaram queda significativa das taxas de infestação para malófagos. BAUM (1968), em contrapartida, verificou uma redução de 85\% na carga de malófagos durante a estação de troca de penas em Turdus merula Linnaeus, 1758 (Turdidae).

Considerando a importância do parasitismo na saúde e conservação das aves e a hipótese de que existe taxa de infestação diferenciada entre a estação reprodutiva e de troca de penas, este trabalho teve como objetivo avaliar a intensidade de parasitismo em Turdus leucomelas Vieillot, 1818 no Parque Estadual do Rio Preto, Minas Gerais, Brasil. Assim, nesse estudo realizamos um levantamento qualitativo de ácaros (Acari: Astigmata e Prostigmata) e quantitativo e qualitativo de malófagos (Insecta: Phthiraptera) desse ambiente, e comparamos a taxa de infestação de malófagos entre a estação reprodutiva e de troca de penas.

\section{MATERIAL E MÉTODOS}

As coletas foram realizadas no Parque Estadual do Rio Preto (18 $\left.07^{\prime} 12,9^{\prime \prime} \mathrm{S}, 43^{\circ} 20^{\prime} 36,9^{\prime \prime} \mathrm{W}\right)$, localizado no município de São Gonçalo do Rio Preto a 355 km a nordeste de Belo Horizonte, na bacia do Rio Jequitinhonha, inserido no complexo da Serra do Espinhaço, no estado de Minas Gerais, Brasil. Com altitudes que variam entre 750 e $1825 \mathrm{~m}$ o parque possui como cobertura vegetal nativa os campos de altitude, os campos rupestres, os cerrados, os cerradões e as matas de altitude, tipologias vegetacionais que cobrem mais de 99,5\% da área (IEF 2006). O clima é marcado por temperaturas cujas médias anuais giram em torno dos $18^{\circ} \mathrm{C}$; a média das máximas é de aproximadamente $27,8^{\circ} \mathrm{C}$ em janeiro, onde concentra a maior quantidade de chuva (307 mm); enquanto que a média das mínimas encontra-se próximo dos $11^{\circ} \mathrm{C}$ em julho, o mês que registra menor índice pluviométrico.

A ave objeto de estudo deste trabalho, T. leucomelas, é encontrada em ambiente semi-florestal e tem preferência pelas bordas de mata (SICK 1997). De hábito alimentar onívora, tem preferência por frutos, mas alimenta-se muitas vezes de insetos que captura revirando folhas no solo (SICK 1997). Tem ampla distribuição geográfica e é encontrada facilmente na área de estudo, motivo da sua escolha.

As coletas foram realizadas em outubro e dezembro de 2006 e março de 2007. As aves foram capturadas com redes de neblina de 10 × 2,5 m, no período compreendido entre a alvo- rada e às 12 horas, num esforço amostral de 789 horas/rede. Todas as aves receberam anilhas metálicas CEMAVE/IBAMA.

A definição das estações reprodutiva e de muda de penas foi realizada considerando aspectos biológicos das aves (MAIAGouvêA et al. 2005). Para a estação reprodutiva foram consideradas somente aves com presença de placa incubatória, segundo orientação do Manual de Anilhamento do CEMAVE (IвAMA 1994). Foram consideradas como plenamente na estação de muda de penas as aves que apresentaram mudas simétricas nas asas, muda das penas do contorno (cabeça, dorso e ventre) simultaneamente com muda das penas das asas, ou muda das penas do contorno simultaneamente com muda das penas da cauda (MARINI \& DuRÃEs 2001).

Ectoparasitos foram coletados utilizando a técnica de "dust-ruffling", que consiste no borrifamento de inseticida em pó à base de piretróide (WaLther \& CLAYTON 1997). O piretróide não é tóxico para as aves (CLAYTON \& TOMPKINs 1995) e garante a coleta de praticamente todos os ectoparasitos permitindo uma análise quantitativa mais precisa (ClaYton \& Drown 2002). Além disso, por ser uma metodologia bastante utilizada, os resultados podem ser comparados com outros trabalhos (e.g. Lindell et al. 2002). A coleta de ectoparasitos foi realizada somente na primeira captura das aves, pois o efeito prolongado do inseticida poderia influenciar nas taxas de infestação das aves recapturadas.

Para ácaros, no entanto, o método não é o mais adequado pelo fato deles se aderirem à raque das penas, sendo necessário, na maioria das vezes, o sacrifício do animal para seu estudo quantitativo. Por este motivo, apenas análises qualitativas foram feitas.

Os malófagos (tombo CAVAISC/IOC-PHT 122-133) assim como os ácaros de pena (tombo CAVAISC/IOC-ACA 352-355) foram armazenados em álcool a 70\% e encontram-se depositados na Coleção de Insetos do Laboratório de Ixodes no Instituto Oswaldo Cruz, Rio de Janeiro, para estudos posteriores.

Para investigar as taxas de infestação, foram realizadas análises como prevalência, dominância, intensidade relativa e intensidade média segundo Bush et al. (1997).

Os índices de similaridade de Morisita-Horn e de diversidade de Shannon-Winner (MagurRan 2003) foram calculados tanto para a estação reprodutiva quanto para a estação de muda de penas; comparações entre as duas estações foram realizadas através do teste T-student (ZAR 1998).

O teste de normalidade de Anderson-Darling foi realizado e como foi constatada a não normalidade dos dados, análises estatísticas não-paramétricas foram realizadas para a comparação entre as infestações parasitárias em cada fase do estudo (reprodução e muda - Friedman com post-hoc de Tukey) e para a comparação das intensidades de parasitismo de cada espécie entre as duas fases de estudo (reprodução e muda - MannWhitney) (ZAR 1998). Os testes estatísticos foram conduzidos ao nível de significância de 5\% e foram realizados pelos programas Minitab 12 e Bioestat 4.0 (Aires et al. 2005). 


\section{RESULTADOS}

Foram capturados 30 indivíduos de T. leucomelas em estágio reprodutivo ou de muda de penas. Nas campanhas de outubro e dezembro de 2006 foram capturados 18 indivíduos em estágio reprodutivo, na campanha de março de 2007 foram capturados 12 indivíduos em estágio de muda de penas.

\section{Análise da infestação por malófagos}

Foram coletados 552 malófagos na estação reprodutiva e 291 na estação de troca de penas; os exemplares coletados pertenciam a Menoponidae e Philopteridae (subordens Amblycera e Ischnocera, respectivamente) e cinco gêneros Menacanthus Neumann, 1912 e Myrsidea Waterston, 1915 pertencentes à Menoponidae e Brueelia Kéler, 1936, Sturnidoecus Eichler, 1944 e Philopterus Nitzsch, 1818 à Philopteridae.

Foi coletado um indivíduo macho de Philopterus sp. em uma ave na estação reprodutiva. Embora os turdídeos sejam também infestados por espécies desse gênero, o morfotipo do único espécime coletado é semelhante aos que infestam aves da família Icteridae, diferindo significativamente daqueles encontrados nos turdídeos (M.P. Valim, Laboratório de Ectoparasitos, Universidade Federal de Minas Gerais, com. pess.). Por isso, esse achado foi considerado como um caso de parasitismo em hospedeiro atípico ou "straggling" (Rózsa 1993).

Tanto na estação reprodutiva como na estação de muda os malófagos mais dominantes foram Myrsidea sp. (57,2 e 69,4\%, respectivamente) e Brueelia sp. (37,3 e 20,0\%, respectivamente). Sturnidoecus sp. apresentou baixa dominância na estação de reprodução $(4,3 \%)$ e significativo aumento na estação de muda de penas $(10,6 \%)$. A dominância de Menacanthus eurysternus Burmeister, 1838 foi baixa na estação reprodutiva $(1,1 \%)$ e na estação de muda de penas este parasito não foi coletado (Tab. I).Considerando todas as aves capturadas em ambas as estações, uma alta prevalência de malófagos foi encontrada $(96,8 \%)$. O malófago mais prevalente na estação reprodutiva foi Myrsidea sp. $(88,9 \%)$ e na estação de muda foi Brueelia sp. (83,3\%). O menos prevalente foi M. eurysternus que esteve presente em $11,1 \%$ das aves no período reprodutivo e não foi encontrado na estação de muda. Entre os malófagos coletados na estação de muda Sturnidoecus sp. apresentou a mais baixa prevalência (33\%) (Tab. I).

Tabela I. Dominância e prevalência de malófagos coletados em $T$. leucomelas nas estações reprodutiva e de troca de penas (outubro e dezembro 2006/março 2007) no Parque Estadual do Rio Preto.

\begin{tabular}{lcccccc}
\hline \multirow{2}{*}{ Malófagos } & \multicolumn{2}{c}{ Dominância (\%) } & & \multicolumn{2}{c}{ Prevalência (\%) } \\
\cline { 2 - 3 } \cline { 6 - 7 } & Reprodução & Muda & & Reprodução & Muda \\
\hline M. eurysternus & 1,1 & 0 & & 11,1 & 0 \\
Myrsidea sp. & 57,2 & 69,4 & & 88,9 & 66,7 \\
Sturnidoecus sp. & 4,3 & 10,6 & & 22,2 & 33,3 \\
Brueelia sp. & 37,3 & 20,0 & & 83,3 & 83,3 \\
\hline Total & 100 & 100 & & - & - \\
\hline
\end{tabular}

As intensidades relativas de Myrsidea sp., Brueelia sp. e Sturnidoecus sp. não diferiram entre as estações reprodutiva e de muda (Tab. II). No entanto, houve diferença nas intensidades relativas entre os malófagos encontrados em T. leucomelas, sendo Myrsidea sp. e Brueelia sp. mais abundantes que os demais em ambas as estações (Tab. III).

Tabela II. Comparação entre as intensidades relativas de cada malófago observado em $T$. leucomelas entre a estação reprodutiva e de troca de penas (outubro e dezembro 2006/março 2007) no Parque Estadual do Rio Preto. ( $\left.{ }^{*}\right)$ Número médio de malófagos por ave, $(\mathrm{N})$ número total de aves amostradas, $\alpha=5 \%, \mathrm{Gl}=1$.

\begin{tabular}{lcccc}
\hline \multicolumn{1}{c}{ Malófagos } & $\begin{array}{c}\text { Reprodução* } \\
\left(\mathrm{N}_{\text {aves }}=18\right)\end{array}$ & $\begin{array}{c}\text { Muda* } \\
\left(\mathrm{N}_{\text {aves }}=12\right)\end{array}$ & $\begin{array}{c}\text { Mann- } \\
\text { Whitney }\end{array}$ & $\begin{array}{c}\text { Valor de } \\
\mathrm{p}\end{array}$ \\
\hline M. eurysternus & 0,33 & - & - & \\
Myrsidea sp. & 17,56 & 16,83 & 294 & 0,54 \\
Brueelia sp. & 11,44 & 4,83 & 317 & 0,11 \\
Sturnidoecus sp. & 1,33 & 2,58 & 265 & 0,46 \\
\hline
\end{tabular}

As maiores intensidades médias de parasitismo, nas aves em estágio reprodutivo, foram observadas para Myrsidea sp. $(19,8)$ e Brueelia sp. $(13,7)$, os parasitos Sturnidoecus sp. $(6,0)$ e M. eurysternus $(3,0)$ apresentaram menor intensidade de infestação neste período. Para as aves em estágio de muda de penas Myrsidea sp. também foi o malófago mais abundante $(25,2)$ seguido por Sturnidoecus sp. $(7,7)$. O parasito Brueelia sp. apresentou menor intensidade de infestação neste período $(5,8)$ e M. eurysternus sequer foi encontrado (Tab. IV).

Os valores de diversidade foram semelhantes para a estação reprodutiva $\left(\mathrm{H}^{\prime}=0,88\right)$ e estação de muda $\left(\mathrm{H}^{\prime}=0,81\right)$. A similaridade entre as estações mostrou-se alta segundo o índice de Morisita-Horn $(0,95)$.

A comparação da intensidade média de malófagos entre as estações, apresentou resultado estatisticamente não significativo ( $\mathrm{W}=30,00 ; \mathrm{gl}=1 ; \mathrm{p}=0,68)$. O número total de ectoparasitos ( $\mathrm{t}=0,69 ; \mathrm{p}=0,50)$ e o número de ninfas $(\mathrm{t}=0,57 ; \mathrm{p}=0,57)$ não apresentaram diferenças estatísticas significativas entre as duas estações, porém o número de adultos de ectoparasitos apresentou um valor significativamente maior na estação reprodutiva ( $\mathrm{t}=2,04 ; \mathrm{p}=0,05$ ) (Fig. 1).

\section{Análise de infestação de ácaros}

Das duas subordens de ácaros coletadas (Astigmata e Prostigmata), Astigmata foi a mais representada, tanto em número de famílias quanto de gêneros, sendo encontrados para esta subordem os seguintes parasitos: Analges sp. (Analgidae), Trouessartia sp. (Trouessartidae) e Proctophyllodes sp. e Pterodectes sp. (Proctophyllodidae).

Para a subordem Prostigmata, foram encontrados apenas indivíduos infestados por ácaros Trombiculidae. A prevalência de trombiculídeos foi de $22 \%$ na estação reprodutiva e $66 \%$ na estação de muda. 
Tabela III. Comparação entre as intensidades relativas dos malófagos observados em $T$. leucomelas nas estações de reprodução e troca de penas (outubro e dezembro 2006/março 2007) no Parque Estadual do Rio Preto (média \pm erro padrão, Gl = 3, $\alpha=5 \%$.).

\begin{tabular}{lcccccc}
\hline \multirow{2}{*}{ Estações } & \multicolumn{4}{c}{ Malófagos } & \multirow{2}{*}{ Friedman } & \multirow{2}{*}{ Valor de $\mathrm{p}$} \\
\cline { 2 - 5 } & M. eurysternus & Myrsidea sp. & Brueelia sp. & Sturnidoecus sp. & \\
\hline Reprodução & $0,33 \pm 0,28$ & $17,56 \pm 4,49$ & $11,44 \pm 2,79$ & $1,33 \pm 0,85$ & 37,03 & $<0,001$ \\
Muda & - & $16,83 \pm 6,42$ & $4,83 \pm 1,42$ & $2,58 \pm 1,52$ & 18,32 & $<0,001$ \\
\hline
\end{tabular}

Tabela IV. Intensidade média de infestação dos malófagos encontrados em $T$. leucomelas nas estações reprodutiva e de troca de penas (outubro e dezembro 2006/março 2007) no Parque Estadual do Rio Preto. (N) Número de indivíduos coletados.

\begin{tabular}{lcccccc}
\hline \multirow{2}{*}{ Malófagos } & \multicolumn{3}{c}{ Reprodução $(\mathrm{N}=18)$} & \multicolumn{3}{c}{ Muda $(\mathrm{N}=12)$} \\
\cline { 2 - 8 } & Ectoparasitos & Aves infestadas & Intensidade infestação & Ectoparasitos & Aves infestadas Intensidade infestação \\
\hline M. eurysternus & 6 & 2 & 3,0 & 0 & 0 & 0 \\
Myrsidea sp. & 316 & 16 & 19,8 & 202 & 8 & 25,2 \\
Brueelia sp. & 206 & 15 & 13,7 & 58 & 31 & 4 \\
Sturnidoecus sp. & 24 & 4 & 6,0 & & 4,7 \\
\hline
\end{tabular}

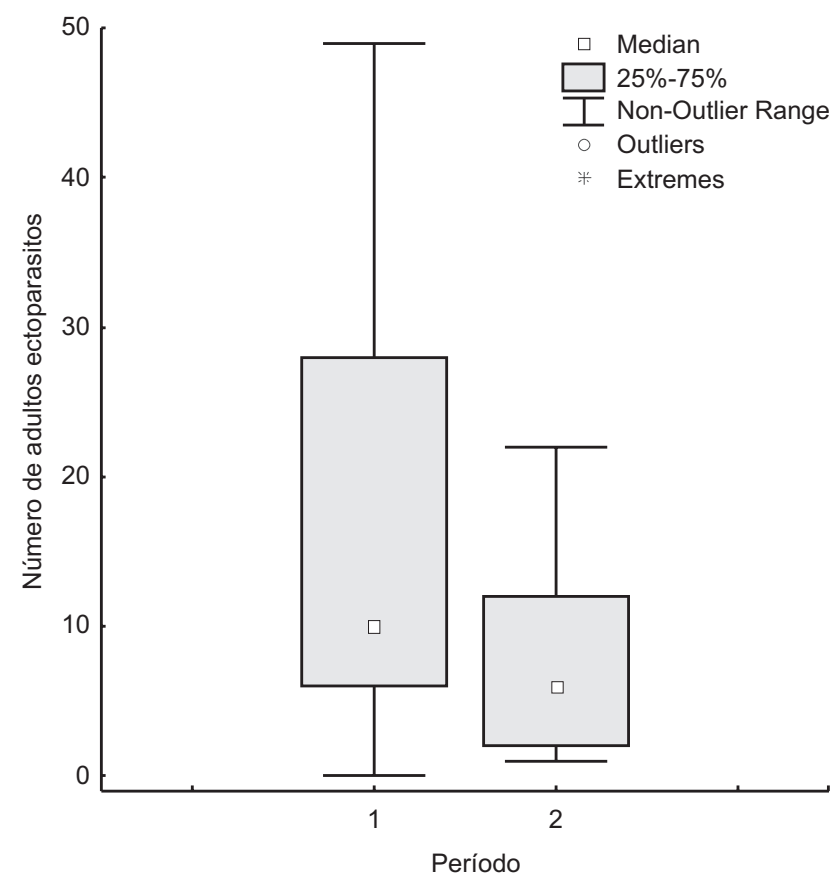

Figura 1. Número total de malófagos adultos (Insecta, Phthiraptera) em Turdus leucomelas (Passeriformes, Turdidae) na estação reprodutiva (1) (outubro e dezembro 2006) e estação de troca de penas (2) (março 2007) no Parque Estadual do Rio Preto, Minas Gerais, Brasil.

Os ácaros de pena (Astigmata) apresentaram altas taxas de prevalência, com $94,4 \%$ de aves infectadas na estação reprodutiva e $100 \%$ durante a muda de penas. Considerando todas as aves capturadas em ambas as estações a prevalência foi de $96,6 \%$.

Os ácaros de pena mais prevalentes foram Analges sp. (83,33\%) na estação reprodutiva e Trouessartia sp. (83,33\%) na estação de muda (Tab. V).

\section{DISCUSSÃO}

\section{Malófagos}

Considerando a alta similaridade encontrada entre as duas estações, os valores de diversidade semelhantes e que não foram encontradas diferenças significativas no número total de malófagos e ninfas para as duas estações, conclui-se que a hipótese de ciclo reprodutivo sincronizado, assim como a redução da carga parasitária na estação de muda de penas, não ocorreu com T. leucomelas na área estudada.

As aves apresentaram alta taxa de prevalência por malófagos no Cerrado do Parque Estadual do Rio Preto (96,8\%). Lyra-Neves et al. (2005) encontraram uma prevalência de 31,9\% de aves (Emberizidae) infestadas por esses ectoparasitos na Mata Atlântica, através de observação simples e catação manual para obtenção dos malófagos. MARINI et al. (1996), também na Mata Atlântica e realizando catação manual, encontraram prevalência de 95,5\% de malófagos em aves Turdidae. A alta prevalência encontrada neste trabalho pode ser explicada pelo método utilizado ("dust-ruffling"), que aumenta consideravelmente a eficácia da coleta de ectoparasitos (Clayton \& Drown 2002). Lindell et al. (2002) encontraram prevalência de 93\% ( $\mathrm{n}=14)$ em Turdus grayi Bonaparte, 1838 e de $86 \%$ para Turdus assimilis Cabanis, $1850(\mathrm{n}=22)$ na Costa Rica, utilizando o mesmo método empregado neste trabalho.

Indivíduos de dieta insetívora e onívora podem apresentar maior prevalência por estarem mais sujeitos ao contato com 
Tabela V. Prevalência de ácaros encontrados em T. leucomelas nas estações reprodutivas e de troca de penas (outubro e dezembro 2006/março 2007) no Parque Estadual do Rio Preto. (N) Número de indivíduos coletados.

\begin{tabular}{|c|c|c|c|c|}
\hline \multirow{2}{*}{ Ácaros } & \multicolumn{2}{|c|}{ Reprodução (n = 18) } & \multicolumn{2}{|c|}{ Muda $(n=12)$} \\
\hline & Aves infestadas & Prevalência (\%) & Aves infestadas & Prevalência (\%) \\
\hline \multicolumn{5}{|l|}{ Astigmata } \\
\hline \multicolumn{5}{|l|}{ Analgidae } \\
\hline Analges sp. & 15 & 83,33 & 10 & 83,33 \\
\hline \multicolumn{5}{|l|}{ Trouessartiidae } \\
\hline Trouessartia sp. & 10 & 55,55 & 10 & 83,33 \\
\hline \multicolumn{5}{|l|}{ Proctophyllodidae } \\
\hline Proctophyllodes sp. & 8 & 44,44 & 4 & 33,33 \\
\hline Pterodectes sp. & 5 & 27,78 & 1 & 8,33 \\
\hline \multicolumn{5}{|l|}{ Prostigmata } \\
\hline Trombiculidae & 4 & 22,22 & 8 & 66,66 \\
\hline
\end{tabular}

ectoparasitos, pois freqüentam diferentes estratos da vegetação durante a procura do alimento, o que aumenta as possibilidades de contato com os parasitos (Pruett-Jones \& Pruett-Jones 1991, Marini et al. 1996, Marini \& Couto 1997). Assim, a dieta onívora de T. leucomelas (Sıck 1997) pode ter influenciado em seus altos índices de infestação.

A diversidade de malófagos que parasitam T. leucomelas no Parque Estadual do Rio Preto varia de moderada a alta e as espécies de ectoparasitos são similares entre as estações de reprodução e muda.

Foster (1969), estudando 2205 indivíduos de Vermivora celata Say, 1823 (Parulidae) depositados em museu, encontrou um pico na produção de ovos de Menacanthus sp. no período pré-reprodutivo. A sincronização do ciclo reprodutivo entre parasito e hospedeiro foi relacionada à maior taxa de hormônios reprodutivos no sangue das aves. Isso indicaria o melhor momento para reprodução dos ectoparasitos, dada a maior possibilidade de transmissão de ninfas para a prole do hospedeiro. Esta hipótese de ciclo sincronizado também foi relatada para aves marinhas (Charadriiformes: Alcidae) devido à alta prevalência de ninfas em aves jovens (Eveleigh \& Threfall 1976).

Foster (1969), também encontrou baixa dominância de Menacanthus sp. na estação de muda, não tendo sido encontrados adultos, ninfas ou ovos deste malófago. O mesmo ocorreu neste trabalho para T. leucomelas com a baixa dominância deste parasito na estação reprodutiva e ausência durante a muda de penas das aves. As espécies de Menacanthus vivem na pele das aves, e não nas penas, como os Philopteridae, desta forma, podemos concluir que a troca de penas não é um mecanismo eficaz para controle destes ectoparasitos. Assim, a maior dominância na estação reprodutiva poderia reforçar a hipótese de ciclo reprodutivo sincronizado para esta espécie, contudo testes diretos devem ser feitos para elucidar esta questão.

Neste trabalho não foi observado aumento significativo no número de ninfas dos malófagos encontrados em T. leucomelas durante a estação reprodutiva. Todavia, outros fatores mais difíceis de serem testados também podem influenciar nas taxas de infestação e ciclo biológico dos ectoparasitos entre as diferentes estações. Dentre eles está a habilidade individual de limpeza das penas ("preening") (Clayton 1991), a saúde das aves (Clayton 1990) e até mesmo fatores abióticos, como a diferença de umidade na localidade onde a ave vive (MOYER et al. 2002).

\section{Ácaros de pena (Astigmata) e trombiculídeos (Prostigmata)}

Os ácaros Astigmata foram os mais prevalentes tanto na estação reprodutiva como na estação de muda de penas. As famílias encontradas parasitam especialmente aves e apesar das altas prevalências aparentemente não apresentaram danos às penas dos T. leucomelas no Parque Estadual do Rio Preto, contudo avaliações diretas devem ser conduzidas para se concluir sobre esta questão. De acordo com Blanco et al. (2001) os ácaros plumícolas se alimentam de detritos e secreções encontrados nas penas. Desta forma, alguns autores consideram que não existe uma relação de parasitismo e sim de comensalismo entre ácaros de pena e aves (GAUd \& ATYEo 1996).

Apenas Analges spp. apresentaram alta prevalência na estação reprodutiva e na estação de muda de penas (Tab. V), o que pode indicar que o mecanismo de troca de penas, em T. leucomelas, não é eficiente para a diminuição da carga parasitária destes ácaros. Este gênero de ácaro não foi encontrado por KANEGAE et al. (2008) em seu estudo com aves do cerrado do Brasil infestando T. leucomelas e, quando encontrado em Turdus spp., apresentou-se com baixa prevalência. JovAni \& SERRANo (2001) sugeriram que os Astigmata possuem um comportamento adaptativo que os permitem identificar a próxima pena a cair no evento de muda, deslocando-se para outro micro-habitat.

A prevalência das espécies de todos os outros gêneros de ácaros variou entre a estação reprodutiva e de muda de penas, sendo Proctophyllodes spp. e Pterodectes spp. mais prevalentes na estação reprodutiva e Trouessartia spp. e espécies de Trombiculidae 
mais prevalentes na estação de muda. Trouessartia spp. e Pterodectes spp. foram os ácaros mais encontrados por KANEgAE et al. (2008) em T. leucomelas, enquanto Proctophyllodes spp. foi pouco encontrado. Segundo Marini et al. (1996), os períodos de maior infestação de ácaros são os mais quentes do ano, e estes períodos podem ou não coincidir com o período reprodutivo das espécies. Neste estudo, o período mais quente e úmido do ano corresponde ao período reprodutivo, onde a infestação por Proctophyllodes spp. e Pterodectes spp. foi maior, corroborando os resultados de MARINI et al. (1996).

Ácaros Trombiculidae foram mais prevalentes na estação de muda, período do outono, onde a umidade e temperatura começam a declinar até atingirem os menores índices no inverno. Este mesmo resultado foi observado por LiteraK et al. (2007) na República Tcheca.

Ácaros de pena podem sofrer influências ambientais que interferem na dinâmica sazonal de infestação, como umidade, temperatura e altitude (MARINI et al. 1996, RoDA \& FARIAS 2007) e estes fatores podem ser os responsáveis por esta diferença e merecem serem testados.

Ácaros hematófagos trombiculídeos, foram encontrados em T. leucomelas, sendo mais frequentes na estação de muda. A troca de penas parece não ser um mecanismo válido para diminuição da carga parasitária destes ácaros visto que eles não vivem nas penas e sim fixados à pele das aves. Ninfas e adultos desta família são de vida livre e somente as larvas são parasitos obrigatórios de aves, répteis e mamíferos, onde podem causar dermatites, chamadas de trombidiose (KRANTZ 1978). Apesar da alta virulência destes parasitos pouco se sabe sobre as taxas de mortalidade e qual a consequência desta dinâmica no controle populacional das aves neotropicais, o conhecimento destes fatores poderiam servir como subsídio para a conservação de aves.

\section{AGRADECIMENTOS}

Agradecemos a Michel P. Valim, Departamento de Parasitologia, Universidade Federal de Minas Gerais, pela identificação dos ectoparasitos; a Felipe C. Ribeiro pela ajuda nos trabalhos de campo; ao Curso de Ecologia da Universidade de Belo Horizonte pela oportunidade de realização deste estudo; ao Instituto de Florestas de Minas Gerais e Antônio A. Tonhão, administrador do Parque Estadual do Rio Preto, pela autorização de uso das dependências do Parque; a Rogério P. Martins pelo apoio na liberação da infra-estrutura do Laboratório de Ecologia e Comportamento de Insetos, Universidade Federal de Minas Gerais.

\section{LITERATURA CITADA}

Aires, M.; M. Ayres-Jr; D.L. Ayres \& A.S. Santos. 2005. BioEstat 4.0: aplicações estatísticas nas áreas das ciências biológicas e médicas. Belém, Ed. Sociedade Civil Mamirauá, MCT, Imprensa Oficial do Estado do Pará, 323p.

BAUM, H. 1968. Biology and ecology of the feather lice of blackbirds. Angew Parasitology 9: 129-175.
Blanco, G.; J.L. Tella; J. Potti \& A. Baz. 2001. Feather mites on birds: cost of parasitism or conditional outcomes? Journal of Avian Biology 32: 271-274.

Bush, A.O.; K.D. Lafferty; J.M. Lotz \& A.W. Shostak. 1997. Parasitology meets ecology on its own terms: Margolis et al. revisited. The Journal of Parasitology 83 (4): 575-583.

Chicchino, A.C. \& M.P. Valim. 2008. Three new species of Formicaphagus Carriker, 1957 (Phthiraptera, Ischnocera, Philopteridae), parasitic on Thamnophilidae and Conopophagidae (Aves, Passeriformes). Zootaxa 1949: 37-50.

Clark, L. 1991. The nest protection hypothesys: The adaptive use of plant secondary compounds by European Starlings, p. 205-221. In: J.E. LoYE \& M. ZuK (Eds). Bird-parasite interactions: ecology, behavior and evolution. Oxford, Oxford University Press, 424p.

Clayton, D.L. 1990. Mate choice in experimentally parasited Rock Doves: Lousy males lose. American Zoologist 30: 251-262.

Clayton, D.L. 1991. Coevolution of avian grooming and ectoparasite avoidance, p. 258-289. In: J.E. LoYE \& M. Zuk (Eds). Bird-parasite interactions: ecology, behavior and evolution. Oxford, Oxford University Press, 424p.

Clayton, D.H. \& D.M. Drown. 2002. Critical evaluation of five methods for quantifying chewing lice (Insecta: Phthiraptera). Journal of Parasitology 87 (6): 1291-1300.

Clayton, D.H. \& D.M. Tompkins. 1995. Comparative effects of mites and lice on the reproductive success of rock doves (Columba livia). Parasitology 110: 195-206.

Clayton, D.H. \& J. Moore. 1997. Host-parasite evolution: general principles and avian models. Oxford, Oxford University Press, 473p.

DufFy, D.C. 1983. The ecology of tick parasitism on densely nesting Peruvian seabirds. Ecology 64: 110-119.

EMLEN, J.T. 1985. Response of breeding cliff swallows to nidicolous parasite infestation. The Condor 88: 110-111.

Eveleigh, E.S. \& W. Threfall. 1976. Population dynamics of lice (Mallophaga) on auks (Alcidae) from Newfoundland. Canadian Journal of Zoology 54: 1694-1711.

Foster, M. 1969. Synchronized life cycles in the orange-crowned warbler and its mallophagan parasites. Ecology 50 (2): 315323.

GaUd, J. \& W.T. Aтуеo. 1996. Feather mites of the world (Acarina, Astigmata): the supraspecific taxa. Part I. Annales Musée Royal de L'Afrique Centrale, Sciences Zoologiques 277: 3-193.

Hernandes, F.A. \& M.P. Valim. 2005. A new species of Pterodectes, Robin, 1877 (Proctophillodidae: Pterodectinae) from the pale-breasted-thrush, Turdus leucomelas (Passeriformes: Turdidae). Zootaxa 1081: 61-68.

Hernandes, F.A. \& M.P. Valim. 2006. Two new species of the feather mite subfamily Pterodectinae (Acari: Astigmata: Proctophillodidae) from Brazil. Zootaxa 1235: 49-61.

Iвама. 1994. Manual de anilhamento de aves. Brasília.Available online at: http://www.ibama.gov.br/cemave/index.php?id_ menu=308 [Accessed: 19/VIII/2008] 
Ief. 2008. Parque Estadual do Rio Preto. Instituto Estadual de Florestas de Minas Gerais. Available online at: http:// www2.ief.mg.gov.br/parques/riopreto/riopreto.asp [Accessed: 14/VII/2008]

Johnson, K.P. \& D.H. Clayton. 2003. The biology, ecology and evolution of chewing lice, p. 449-476. In: R.D. PricE; R.A. Hellemhal; R.L. Palma; K.P. Johnson \& D.H. Clayton (Eds). The Chewing Lice: word checklist and biological overview. Illinois Natural History Survey Special Publication, 24, $\mathrm{XX}+501 \mathrm{p}$.

Jovani, R. \& D. Serrano. 2001. Feather mites (Astigmata) avoid moulting wing feather of passerine birds. Animal Behaviour 62 (4): 723-727.

Kanegae, M.F.; M. Valim.; M.A. Fonseca, M.A. Marini \& N.M.S. Freire. 2008. Ácaros plumícolas (Acari: Astigmata) em aves do cerrado do Distrito Federal, Brasil. Biota Neotropical 8 (1): 31-39.

KrantZ, G.W. 1978. A Manual of Acarology. Corvallis, Oregon State University Book Stores, XX+335p.

LEHANE, M.J. 1991. Biology of blood-sucking insects. London, Harper Collins, 336p..

Lindell, C.A.; T.A. Gavin; R.D. Price \& A.L. SAnders. 2002. Chewing louse distributions on two Neotropical thrush species. Comparative Parasitology 69 (2): 212-217.

Literak, I.; E. Kocianova; F. Dusbabek; J. Martinu; P. Podzemny \& O. Sychra. 2007. Winter infestation of wild birds by ticks and chiggers (Acari: Ixodidae, Trombiculidae) in the Czech Republic. Parasitology Research 101 (6): 1709-1711.

Loye, J.E. \& S.P. Carrol. 1991. The effect of nest ectoparasite abundance on cliff swallow colony site selection, nestling development, and departure time, p. 222-241. In: J.E. LoYE \& M. ZuK (Eds). Bird-parasite interactions: ecology, behavior and evolution. Oxford, Oxford University Press, 424p.

Loye, J.E. \& S.P. Carrol. 1998. Ectoparasite behavior and its effects on avian nest site selection. Annals of Entomology Society of America 91 (2): 159-163.

Lyra-Neves, R.M.; A.M. Farias \& W.R. Telino-Júnior. 2005. Interações entre Phthiraptera (Insecta) e aves (Emberizidae) de Mata Atlântica, Pernambuco, Brasil. Revista Ornithologia 1: 43-47.

Magurran, A.E. 2003. Measuring Biological Diversity. Oxford, Blackwell Publishing, 260p.

Marini, M.Â. \& D. Couto. 1997. Correlações ecológicas entre ectoparasitos e aves de floresta de Minas Gerais, p. 210-218. In: L.L. LeITE \& C.H. SAITO (Eds). Contribuição ao Conhecimento Ecológico do Cerrado. Brasília, Departamento de Ecologia, Universidade de Brasília, 325p.

Marini, M.Â. \& R. Durães. 2001. Annual patterns of molt and reproductive activity of passerines in south-central Brazil.
The Condor 103: 767-775.

Marini, M.Â.; B.L. Reinert; M. Bornschein; J.C. Pinto \& M. Pichorim. 1996. Ecological correlates of ectoparasitism in birds from the Atlantic Forest, Brazil. Ararajuba 4: 93-102.

Marshall, A.G. 1981. The Ecology of Ectoparasitic Insects. London, Academic Press, XVI+459p.

Maya-GouvêA, E.R.; E. GouvêA \& A. Piratelli. 2005. Comunidade de aves de sub-bosque em uma área de ntorno do Parque Nacional do Itatiaia, Rio de Janeiro, Brasil. Revista Brasileira de Zoologia 22 (4): 859-866.

Moyer, B.R.; D.W. Gardiner \& D.H. Clayton. 2002. Impact of feather molt on ectoparasites: looks can be deceiving. Oecologia 131: 203-210.

Paterson, A.M. \& R.D. Gray. 1997. Host-parasite cospeciation, host switching and missing the boat, p. 236-250. In: D.H. Clayton \& J. Moore (Eds). Host-parasite evolution: general principles and avian models. Oxford, Oxford University Press, 488p.

Perrins, C.M. 1965. Population fluctuations and clutch size in the great tit, Parus major L. Journal of Animal Ecology 34: 601-647.

Pruett-Jones, S. \& M.A. Pruett-Jones. 1991. Analysis and ecological correlates of tick burdens in a New Guinea avifauna, p. 154176. In: J.E. LoYE \& M. ZuK. (Eds). Bird-parasite interactions: ecology, behavior and evolution. Oxford, Oxford University Press, 424p.

RodA, S.A. \& A.M.I. Farias. 2007. Ácaros plumícolas em beijaflores no Município de Vicência, Pernambuco, Brasil. Lundiana 8 (1): 13-16.

RózsA, L. 1993. Speciation patterns of ectoparasites and 'straggling' lice. International Journal of Parasitology, 23: 859-864.

Sick, H. 1997. Ornitologia brasileira. Rio de Janeiro, Nova Fronteira, X+912p.

Storni, A.; M.A.S. Alves \& M.P. Valim. 2005. Ácaros de pena e carrapatos (Acari) associados a Turdus albicollis Vieillot (Aves, Muscicapidae) em uma área de Mata Atlântica da Ilha Grande, Rio de Janeiro, Brasil. Revista Brasileira de Zoologia 22 (2): 419-423.

VALIM, M.P. \& F.A. HeRnANDEs. 2006. Redescriptions of four species of the feather mite genus Pterodectes Robins, 1877 (Acari: Proctophillodidae: Pterodectinae) described by Hebert F. Berla. Acarina 14 (1): 41-55.

Walther, B.A. \& D.H. Clayton. 1997. Dust-ruffling: A simple method for quantifying ectoparasite loads of live birds. Journal of Field Ornithology 68: 509-518.

ZAR, J.H. 1998. Biostatistical Analysis. New Jersey, Prentice Hall, $4^{\text {th }}$ ed., 929 p.

Submitted: 13.XI.2008; Accepted: 10.IX.2009.

Editorial responsibility: Marcus Vinicius Domingues 